\title{
O uso do google maps como tecnologia educacional para mapeamento da área no processo de territorialização virtual na atenção básica: um relato de experiência
}

The use of google maps as educational technology for mapping the area in the process of virtual territorialization in basic care: an experience report

El uso de google maps como tecnología educativa para el mapeo del área en el proceso de territorialización virtual en cuidados básicos: informe de experiencia

Ayane Araújo Rodrigues
ORCID: https://orcid.org/0000-0001-7792-6993
Instituto de Educação Superior do Vale do Parnaíba, Brasil
E-mail: ayanerodrigues2012@ hotmail.com
Brenda Ellen Meneses Cardoso
ORCID: https://orcid.org/0000-0002-5982-5985
E-mail: cbrendaellen@ @otmail.com
Victor Brito Dantas Martins
Instituto de Educação Superior do Vale Parnaíba, Brasil
ORCID: https://orcid.org/0000-0002-6932-915X
Secretaria de Saúde de Pimenteiras, Brasil
E-mail: victorbritodantas@gmail.com
Felipe Rodolfo Pereira da Silva
ORCID: https://orcid.org/0000-0001-9224-5571
Universidade Federal do Delta do Parnaíba, Brasil
E-mail: feliperodolfo.15@ hotmail.com
José Osmar Carneiro Alves
ORCID: https://orcid.org/0000-0002-8830-8688
Secretaria de Saúde de Santana do Acaraú, Brasil
E-mail: odontosmar@hotmail.com
André dos Santos Carvalho
ORCID: https://orcid.org/0000-0001-5259-6481
Universidade Federal do Delta do Parnaíba, Brasil
E-mail: fko.andre@gmail.com
Ilana Gomes Soares
ORCID: https://orcid.org/0000-0003-0038-3673
Universidade Federal do Delta do Parnaíba, Brasil
E-mail: mayarastefanni @hotmail.com
Vinícius Duarte Pimentel
E-mail: Luenna.95@gmail.com
ORCID: https://orcid.org/0000-0002-3802-5452
E-mail: viniciusduarttee@gmail.com
Universidade Federal do Delta do Parnaíba, Brasil
Secretaria Municipal de Saúde de Pimenteiras, Brasil
E-mail: Ilana.gomes@ hotmail.com
Maria Luenna Alves Lima
ORCID: https://orcid.org/0000-0003-3409-0078
Secretária Municipal de Saúde de Pimenteiras, Brasil
Mayara Stefanni de Lacerda Bezerra

\section{Resumo}

Objetivo: objetivo demonstrar a importância do uso do Google Maps como recurso didático aplicado na territorialização em saúde de forma virtual. Metodologia: Trata-se de um relato de experiência, o qual descreve reflexões e aspectos vivenciados em relação à aplicação do Google Maps na territorialização em atenção básica. Resultados: A possibilidade oferecida pelo Google maps de realizar a territorialização em saúde de forma virtual, propiciou ao aluno a oportunidade de conhecer o território mesmo em tempos de isolamento social e se mostrou uma ferramenta geotecnológica muito eficaz no processo de territorialização, comtemplando dessa forma todos os objetivos propostos no roteiro para territorialização virtual. O mapeamento e o georreferenciamento, através do Google maps, objetivam tornar o processo de territorialização algo contínuo, favorecendo a aproximação entre profissionais e população. Conclusão: o Google maps tem grande potencial como recurso didático, pois se mostrou 
eficaz promovendo uma aproximação com a realidade espacial e social, contribuindo para um melhor entendimento dos alunos nos aspectos abordados durante processo de territorialização em saúde realizado na atenção básica.

Palavras-chave: Tecnologia educacional; Atenção básica; Área programática de saúde.

\begin{abstract}
Objective: objective to demonstrate the importance of using Google Maps as a didactic resource applied in the territorialization of health in a virtual way. Methodology: This is an experience report, which describes reflections and aspects experienced in relation to the application of Google Maps in the territorialization in primary care. Results: The possibility offered by Google maps to perform territorialization in health in a virtual way, provided the student with the opportunity to know the territory even in times of social isolation and proved to be a very effective geotechnological tool in the process of territorialization, thus contemplating all the objectives proposed in the roadmap for virtual territorialization. The mapping and georeferencing, using Google maps, aim to make the process of territorialization somewhat continuous, favoring the approximation between professionals and the population. Conclusion: Google maps has great potential as a didactic resource, as it proved to be effective in promoting an approximation with the spatial and social reality, contributing to a better understanding of students in the aspects addressed during the process of territorialization in health carried out in primary care.
\end{abstract}

Keywords: Educational technology; Basic attention; Programmatic health area.

\title{
Resumen
}

Objetivo: objetivo demostrar la importancia del uso de Google Maps como recurso didáctico aplicado en la territorialización de la salud de forma virtual. Metodología: Se trata de un relato de experiencia, que describe reflexiones y aspectos vividos en relación a la aplicación de Google Maps en la territorialización en atención primaria. Resultados: La posibilidad que ofrecen los mapas de Google para realizar la territorialización en salud de manera virtual, brindó al alumno la oportunidad de conocer el territorio incluso en tiempos de aislamiento social y demostró ser una herramienta geotecnológica muy efectiva en el proceso de territorialización, por lo que contemplando todos los objetivos propuestos en la hoja de ruta para la territorialización virtual. El mapeo y la georreferenciación, utilizando Google maps, tienen como objetivo hacer que el proceso de territorialización sea algo continuo, favoreciendo la aproximación entre profesionales y población. Conclusión: Google maps tiene un gran potencial como recurso didáctico, ya que proporciona ser efectivo en promover una aproximación con la realidad espacial y social, contribuyendo a una mejor comprensión de los estudiantes en los aspectos abordados durante el proceso de territorialización en salud realizado en atención primaria.

Palabras clave: Tecnología educativa; Atención básica; Área programática de salud.

\section{Introdução}

A pandemia ocasionou um isolamento social em que os alunos estão impedidos de ir assistir as aulas presenciais, o que tornou a educação à distância (EAD) um fator essencial nesse contexto, culminando em uma migração temporária para o ensino digital (Google e IAT, 2020). O Ensino remoto utilizado atualmente no Brasil em situações de emergência é semelhante ao EAD somente no que se refere a uma educação mediada pela tecnologia. No entanto, esses princípios são os mesmos da educação presencial (Rabello, 2020).

A Base Nacional Comum Curricular (2017) traz dentre suas competências gerais: usar o conhecimento da tecnologia e da linguagem digital para se expressar e compartilhar informações, experiências, pensamentos, ideias e sentimentos em diferentes contextos e, com eles, produzir sentidos que levem a compreensão mútua. Ao inserir as novas tecnologias no ensino, os alunos podem expandir sua realidade e conhecimento. Segundo Giordani, Audino e Cassol (2006), entre outros fatores, o processo de melhoria da qualidade do ensino envolve o uso da tecnologia na educação e adota novas metodologias de ensino e aprendizagem. A tecnologia deve ser utilizada como recurso didático por meio de suas possibilidades de interação, simulação, animação e visualização. Para isso, os professores devem estar qualificados para usar bem as ferramentas técnicas (Giordani et al., 2006).

O Google Maps é uma ferramenta técnica educacional porque permite interação com os alunos para que eles possam ser criativos e ter recursos simples de tecnologia de mapeamento. Possui uma interface amigável que permite a interação de sentidos e inteligência, promovendo assim a representação dos alunos no espaço local. É importante a interação porque pode promover a reorganização cognitiva por meio do conflito resultante e, quando o conflito é superado, torna-se conhecimento 
(Fonseca, 2010). Para Coscarelli (1998), bons programas são aqueles que oferecem suporte para a reflexão, incentivam a versatilidade no uso de estratégias e investigam diferenças de interesses pessoais.

Assim, o presente trabalho foi resultado da utilização do Google Maps como recurso didático de representação e análise espacial na territorialização em saúde de forma virtual. Esse serviço permite que os alunos vivenciem várias formas de símbolos e representações, podendo construir e reconstruir mapas com base nos interesses dos alunos, professores e / ou metas estabelecidas (Fonseca, 2010). No Brasil, a territorialização é um propósito básico da Estratégia de Saúde da Família (ESF), sendo implementado pelo Ministério da Saúde desde 1994. Assim, a organização territorial dos serviços ofertados pelo Sistema Único de Saúde (SUS) a qual orienta a delimitação espacial, a fim de direcionar o cuidado no combate às doenças no Território-Área e nos Territórios-Domicílios (Borges \& Taveira, 2018).

Conhecer o território implica um processo de reconhecimento das principais necessidades em saúde daquele espaço geográfico. Por isso, a importância de ter a área de abrangência com a ESF, já que permite a aproximação com perfil demográfico e epidemiológico, contexto histórico e cultural, equipamentos sociais e outros dados relevantes para intervenção no processo saúde-doença da comunidade adscrita (Brasil, 2006).

Desse modo, este trabalho tem como objetivo demonstrar a importância do uso do Google Maps como recurso didático aplicado na territorialização em saúde de forma virtual.

\section{Metodologia}

Trata-se de um relato de experiência, o qual descreve reflexões e aspectos vivenciados em relação à aplicação do Google Maps como tecnologia educativa na territorialização em saúde de forma virtual, realizada no ano de 2020, durante a disciplina Integração Serviço-Ensino-Comunidade II, no Regime Especial de Aprendizagem Remota (REAR), no segundo período do curso de Medicina da Faculdade de Ciências Humanas, Exatas e da Saúde do Piauí/ Instituto de Educação Superior do Vale do Parnaíba (FAHESP/IESVAP), da cidade de Parnaíba-PI. Trata-se de uma perspectiva qualitativa, realizada a partir de métodos descritivos e observacionais. Os métodos qualitativos são aqueles nos quais é importante a interpretação por parte do pesquisador com suas opiniões sobre o fenômeno em estudo (Ludke e Andre, 2013). A observação geralmente é utilizada como uma parte importante no desenvolvimento da pesquisa, é organizada para registrar as informações obtidas durante a sua execução. A vantagem de usar a técnica é que os fatos são percebidos diretamente, sem qualquer intermediação (Pereira A.S. et al., 2018).

Entende-se por integração ensino-serviço como uma estratégia que impulsiona o trabalho coletivo buscando a articulação e integração dos atores envolvidos no âmbito da aprendizagem, promovendo a participação de estudantes e professores, seja na área pedagógica ou saúde em articulação com gestores e profissionais, contribuindo para a melhoria na qualidade do processo formativo dos profissionais de saúde (Mendes et al., 2020).

Metodologicamente o presente trabalho foi constituído de quatro etapas: primeiramente foi solicitado, junto à coordenação da atenção básica, os mapas das áreas de abrangência das ESF; em segundo, realizou-se a sala de aula invertida com estudo prévio sobre o uso do Google Maps; em terceiro, aplicou-se o Google Maps na territorialização das áreas; em quarto, ocorreu a socialização e envio do mapa construído pelos alunos.

\section{Resultados e Discussão}

Durante a etapa de solicitação dos mapas das áreas de abrangência, foi realizada a escolha de 4 áreas para ser analisadas no Google Maps (FIGURAS 1-4). 
Figura 1-2. Áreas para territorialização virtual.

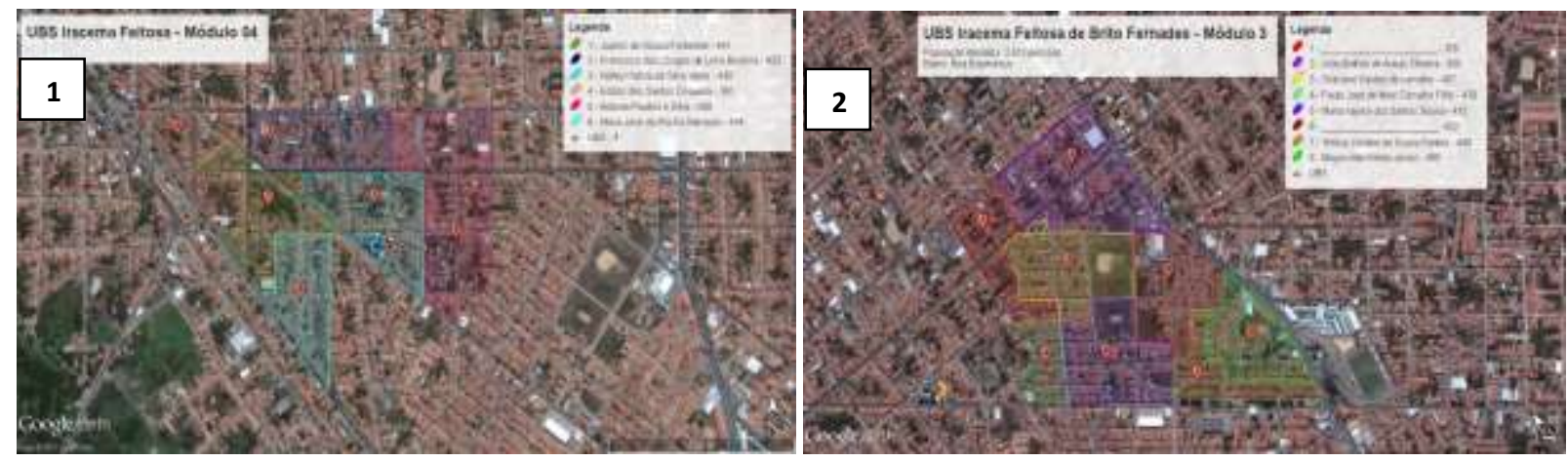

Fonte: Google Maps.

Figura 3-4. Áreas para territorialização virtual.

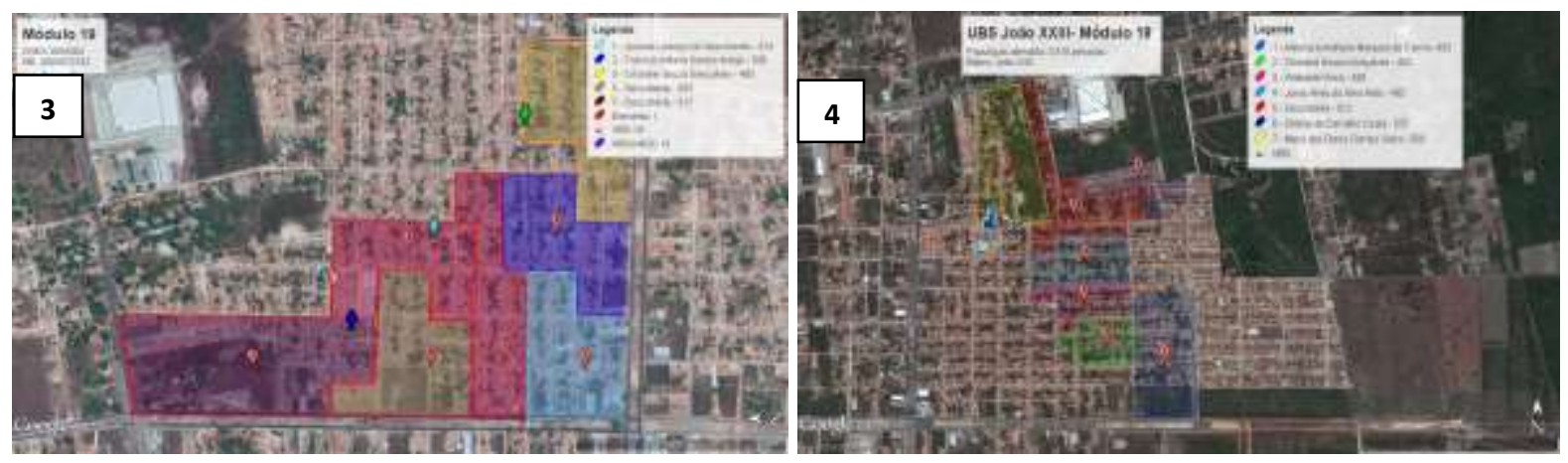

Fonte: Google Maps.

O território - Área é o grupo de microáreas de responsabilidade da equipe de saúde da família (Brasil, 2006). Cada equipe de saúde da família é responsável por monitorar um determinado número de pessoas localizadas em uma determinada área geográfica. É possível planejar e realizar intervenções de atenção à saúde conforme a carência de saúde da comunidade local, bem como as previstas nas protocolos e prioridades da gestão local.

$\mathrm{Na}$ etapa de sala de aula invertida, foram enviados para os alunos artigos para leitura prévia sobre a utilização do Google Maps. A proposta da sala de aula invertida entrelaça vários conceitos: aprendizagem ativa e colaborativa, autonomia do aluno, aprendizagem significativa e os conceitos de aprendizagem / educação a distância. Nesse contexto, o professor atua muito mais como aquele que planeja e implementa a atividade de ensino, atuando como um facilitador da aprendizagem (Bollela \& Cesaretti, 2017).

$\mathrm{Na}$ terceira etapa, os alunos foram divididos em 4 grupos, onde cada grupo recebia o mapa da área de abrangência de uma ESF. Em seguida, a pesquisa no Google Maps foi realizada, seguindo um roteiro para identificar a localização, os limites do território, os equipamentos sociais, áreas de lazer, os pontos de atenção à saúde e a urbanização existente no território (Quadro 1). 
Quadro 1. Roteiro para orientação na territorialização virtual.

\begin{tabular}{|ll|}
\hline$\bullet$ & Pesquisar localização e introduzir no relatório. \\
\hline$\bullet$ & Identificar território: urbana ou rural. \\
\hline$\bullet$ & $\begin{array}{l}\text { Identificar os recursos existentes no território: rede de enfermagem, equipamentos sociais, } \\
\text { áreas de lazer e apresentá-los no mapa; }\end{array}$ \\
\hline$\bullet$ & Observe a urbanização: transporte público, redes viárias, pavimentação. \\
\hline
\end{tabular}

Fonte: Autores.

O endereço para a busca da localização da área no Google Maps constava nas orientações do roteiro. Essa etapa foi previamente realizada pelo docente para que facilitasse a busca correta do aluno durante a aula virtual. Para Giffoni, Barroso e Sampaio (2020), a compreensão sobre os usos de novas tecnologias é de essencial importância para o desenvolvimento progressivo de saberes.

Após a localização da área, os alunos observaram o mapa cedido pela coordenação da atenção básica do município e, assim, observaram e marcaram os limites territoriais, construindo dessa forma um esboço do mapa. Em seguida foi proposta a busca pelos equipamentos sociais presentes no território que são representados por associações dos bairros, igrejas, escolas e creches. Ao mesmo tempo, também foi proposto a busca pelas áreas de lazer e pontos de atenção pertencentes a rede de atenção à saúde. As áreas de lazer são representadas por parques, campos de futebol, quadras de esportes e praças. Os pontos de atenção são representados por hospitais, consultórios, clínicas, laboratórios, rede psicossocial e de urgência e emergência. Após a busca os equipamentos sociais, as áreas de lazer e os pontos de atenção à saúde foram identificados no mapa. Vários aspectos da urbanização também são observados, como transporte público, redes viárias e pavimentação. Por fim, cada grupo de aluno socializou o mapa com as informações que encontraram na área pesquisada (Figura 5).

Figura 5. Mapa Virtual.

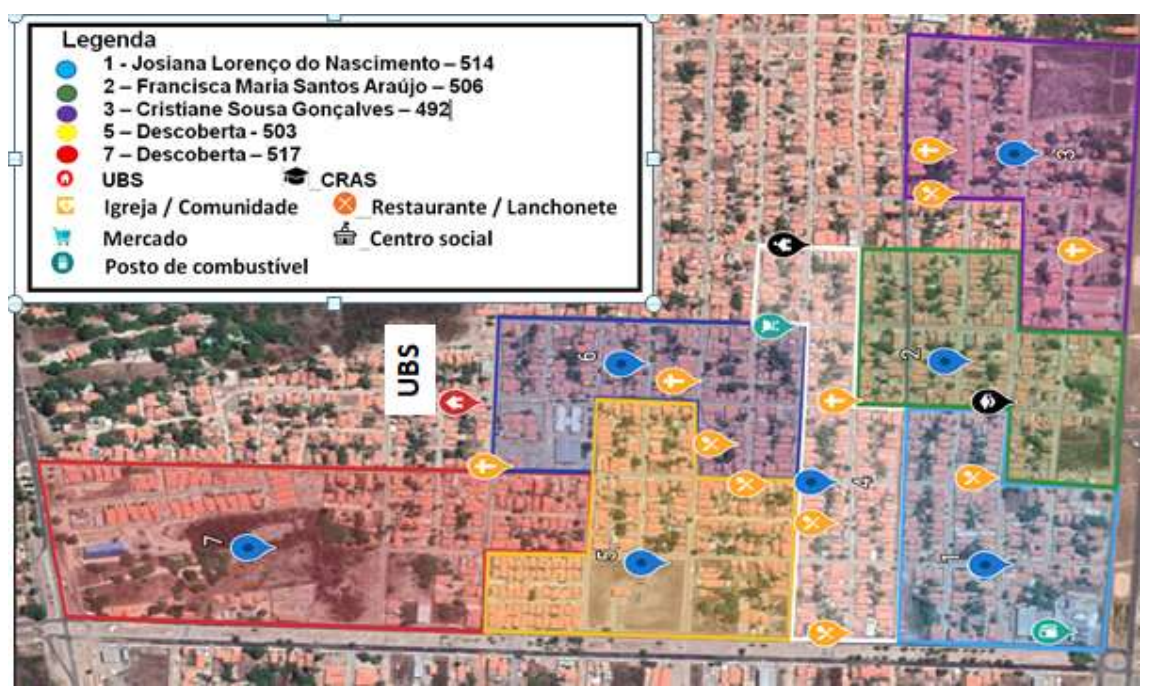

Fonte: Autores.

Durante o processo de utilização do Google Maps, houve maior interação e engajamento dos alunos, assim como maior motivação do docente no mapeamento e conhecimento dos territórios, a fim de localizar e reconhecer os recursos sociais existentes que auxiliam os profissionais de saúde no planejamento das práticas em saúde no território. O processo de 
regionalização da APS pode ser entendido como uma técnica de planejamento e gestão que visa propor intervenções a partir da realidade (Faria, 2013). Para tanto, como tarefa comum a todos os profissionais, a Política Nacional de Atenção Básica (PNAB) 2017 estabeleceu o papel de participar do processo de regionalização para identificar grupos, famílias e indivíduos em risco e vulnerabilidade (Brasil, 2017). Porém, Chaves e Mafra (2004) chamam a atenção para o andamento desse processo, que pode ser facilmente reduzido e simplificado, tornando-se um procedimento burocrático. O professor ao informar o aluno deste programa e incentivá-lo a utilizar, proporcionará a ele uma melhor entendimento do espaço geográfico em suas dimensões físicas, sociais e espaciais (Silva \& Chaves, 2011).

A possibilidade oferecida pelo Google Maps de realizar a territorialização em saúde de forma virtual, propiciou ao aluno a oportunidade de conhecer o território mesmo em tempos de isolamento social e se mostrou uma ferramenta geotecnológica muito eficaz no processo de territorialização, contemplando, dessa forma, todos os objetivos propostos no roteiro para territorialização virtual. O mapeamento e o georreferenciamento, através do Google Maps, objetivam tornar contínuo o processo de territorialização, favorecendo a aproximação entre profissionais e população. Dessa forma, espera-se um melhor entendimento das necessidades de saúde, bem como o planejamento da organização do trabalho, a inclusão de pessoas que não são consideradas prioritárias e o trabalho na rede e no território (Camargos \& Oliver, 2019).

\section{Conclusão}

O Google Maps apresentou, como vantagem, o conhecimento das caraterísticas da área sem a necessidade de ir ao local. Tem como ferramentas o zoom, as imagens dos locais e a visualização dos recursos existentes no território. Como limitações, apresentou mapas de algumas áreas indisponíveis e a desatualização de outras, além de requerer uma certa habilidade manual.

Em vista disso, o Google Maps tem grande potencial como recurso didático, mesmo com as limitações citadas, pois contemplou todos os objetivos propostos na territorialização virtual. É uma tecnologia educacional de grande engajamento e interação entre os alunos e que provoca maior motivação dos decentes durante o uso. A territorialização virtual se mostrou eficaz pois promoveu uma aproximação com a realidade espacial e social, contribuindo para uma melhor compreensão dos alunos nos aspectos abordados durante as etapas de territorialização em saúde realizado na atenção básica.

Finalmente, são necessários mais estudos sobre a aplicabilidade do Google Maps e que proponham novas metodologias no ensino aprendizagem para auxiliar na transmissão dos conteúdos e alcance dos objetivos de aprendizagem.

\section{Referências}

Base Nacional Comum Curricular. (2020). Base Nacional Comum Curricular: Educação e a Base: Ministério da Educação.

Brasil. (2006). Cadernos de Atenção Básica: Ministério da Saúde.

Brasil. (2017). Política Nacional de Atenção Básica: Ministério da Saúde.

Brasil. (2006). Portaria nº 750: Ministério da Saúde.

Bollela V. R., \& Cesaretti, M. L. R. (2017). Sala de aula invertida na educação para as profissões de saúde: conceitos essenciais para a prática. Revista Eletrônica de Farmácia. 14(1), 39-48.

Borges C., \& Taveira, V. R. (2018). Territorialização. Tratado de medicina de família e comunidade: princípios de formação e prática, 2432.

Camargos, M. A., \& Oliver F. C. (2019). Uma experiência de uso do georreferenciamento e do mapeamento no processo de territorialização na Atenção Primária à Saúde. Saúde Debate, Rio de Janeiro, 43(123), 1259-1269.

Chaves, M. M. N., \& Mafra, M. R. P. (2004) O Processo de Territorialização e a Atenção à Saúde no Programa Saúde da Família. Família, Saúde e Desenvolvimento (UFPR), 6(2):127-133.

Coscarelli, C. V. (1998). O uso da informática como instrumento de ensino-aprendizagem. Presença Pedagógica. 4(20), 37-45. 
Research, Society and Development, v. 10, n. 2, e35610212677, 2021 (CC BY 4.0) | ISSN 2525-3409 | DOI: http://dx.doi.org/10.33448/rsd-v10i2.12677

Faria, R. M. (2013). A Territorialização da atenção primária à saúde no Sistema Único de Saúde e a construção de uma perspectiva de adequação dos serviços aos perfis do território. Hygeia - Revista Brasileira de Geografia Médica e da Saúde, 6(16):131-147.

Fonseca, R. A. (2010). Uso do google mapas como recurso didático para mapeamento do espaço local por crianças do ensino fundamental da cidade de Ouro Fino. Tese (doutorado), Universidade Estadual Paulista, Rio Claro.

Giordani, A. C. C., Audino, D. F., \& Cassol, R. (2006). Inserção do Google Earth no ensino de Geografia. 12 Jornada Nacional de Educação/ ${ }^{\circ}$ Congresso Internacional de Educação. Educação e sociedade: perspectivas educacionais no século XXI, 1-8.

Giffoni, J. S., Barroso, M. C. S., \& Sampaio, C. G. (2020). Aprendizagem significativa no ensino de Química: uma abordagem ciência, tecnologia e sociedade. Research, Society and Development, 9(6), e13963416.

Google, I. A. T. (2020). Coronavírus: o mundo nunca mais será o mesmo.https://www.mmaglobal.com/files/328._brazil_1_5010688669204349081.pdf

Ludke, M., \& Andre, M. E. D. A (2013). Pesquisa em educação: uma abordagem qualitativa. (2a ed.): EPU.

Mendes, T. M. C., Ferreira, T. L.S., Carvalho, Y. M., Silva, L. G., Souza, C., et al. (2020). Contributions and challenges of teaching-service-community integration. Texto \& Contexto - Enfermagem, 29, e20180333.

Pereira A. S. et al. (2018). Metodologia da pesquisa científica [recurso eletrônico. UFSM.

Rabello, M. E. (2020). Lições do coronavírus: ensino remoto emergencial não é EAD: https://desafiosdaeducacao.grupoa.com.br/coronavirus-ensino-remoto/

Silva, A. P. A., \& Chaves, J. M. (2011). Utilização do Google Maps e Google Earth no ensino médio: estudo de caso no Colégio Estadual da Polícia MilitarDiva Portela em Feira de Santana-BA. Anais XV Simpósio Brasileiro de Sensoriamento Remoto - SBSR, Curitiba, PR, Brasil. 